\title{
Zirconium Zr 89-labeled Envafolimab
}

National Cancer Institute

\section{Source}

National Cancer Institute. Zirconium Zr 89-labeled Envafolimab. NCI Thesaurus. Code C162860.

A radioimmunoconjug ate composed of envafolimab, a monoclonal antibody directed against programmed cell death-1 ligand 1 (PD-L1; cluster of differentiation 274; CD274) and labeled with the radioisotope zirconium $\mathrm{Zr}$ 89, with potential use for assessing PD-L1 tumor antigen expression using positron emission tomography (PET). Upon administration of zirconium Zr 89-labeled envafolimab, the envafolimab moiety targets and binds to the PD-L1 expressed on certain tumor cells. Upon uptake and PET imaging, the radioisotope moiety can be visualized and the extent of PD-L1 expression can be assessed. This can be used to predict the therapeutic response to envafolimab and other PD-L1-targeting agents. 